Session 2202

\title{
The New Aerospace Engineering Curriculum at The Ohio State University
}

\author{
H. Öz and M. R. Foster \\ Aerospace Engineering Program \\ Department of Aerospace Engineering, Applied Mechanics and Aviation \\ The Ohio State University
}

\section{Introduction and Background}

Our discipline has changed a great deal since the boom years of aeronautical and astronautical engineering in the 1950's and 1960's; apart from obvious changes due to altered geopolitics, there have also been changes in the way engineers do their jobs in the workplace. Perhaps more important to educators, there have been changes in the student culture. The Course of Study for Aeronautical and Astronautical Engineering at The Ohio State University changed significantly once before, in 1970, when the university made a transition from a 5-year B. S. degree to what had become the standard everywhere: the 4-year degree. Since that time, only minimal changes have occurred.

Though the curriculum has remained essentially static, the student world has changed dramatically. Sociologists have thoroughly documented many of those changes; one of the most illuminating is the cultural analysis by $\operatorname{Postman}^{1}$ in a popular account of the cultural shifts due to the impact of television. Of prime concern to engineering educators are two of his points: (1) Knowledge has become fragmented, exemplified in the 'sound bytes' now characteristic of our national life. (2) A society characterized by visual communication as our is, as opposed to print communication as ours used to be, has more difficulty expressing complex ideas accurately; less precision is inherent in the medium. In addition, for a variety of reasons--among them, increased tuition at large state universities, lessened availability of student-aid dollars, and some unwillingness for students to defer material gratification--more and more students work in jobs for 20 or so hours per week. This increased number of working students means that, for many, the "four-year degree" is simply fiction. Five years has become the norm at many public universities. Further, perhaps in part because of less time for study for some due to those work commitments, it is the common observation of many university faculty members that many students, though certainly not all, appear less able to assimilate information learned in a sequence of courses into any kind of integrated whole. Neither specific skills nor general knowledge seem to penetrate course boundaries. Typically, a professor utilizes a tool that has been taught in a prior course to solve a particular engineering problem; students seem mystified and claim they have "never seen it before", a statement at odds with what was in fact taught in the preceding, prerequisite course. Certainly Postman's point about the fragmentation of knowledge may help to explain the phenomenon, but provides no remedies. 
Faced not only with these changes in students, but also with new and more persistent voices from industry about the teaching of design, in particuar ${ }^{2,3,4}$, and a level of dissatisfaction inside academia for some of the reasons already noted, a number of institutions have undertaken significant change in their undergraduate courses of study, with the Michigan 2000 study among the most prominent ${ }^{5}$. A number of themes emerge from such studies, principally these: Design should be integrated more fully through the curriculum, and more emphasis should be given to what have often been called "softer" skills like communications and ethics, for example. The dialogue has led to new accreditation criteria outlined in ABET $2000^{6}$.

Programs in aerospace engineering, more particularly, have also been evolving. Changes are outlined in Michigan $2000^{5}$ for their program. MIT has also recently radically altered the way it teaches undergraduate majors in Aeronautics and Astronautics ${ }^{7}$, again laying more stress on communications, systems engineering and design, and allowing students to choose specialization tracks. The University of Maryland has rethought what aerospace engineering should look like in the future, emphasizing not only more design, but laying more emphasis on control systems and avionics as disciplines of increasing importance ${ }^{8}$. It is evident that aerospace engineering faculties across the country have understood the need for change in the way engineering is taught, rejecting "business as usual" in favor of new paradigms for education.

\section{The Evolution of the New Curriculum}

In our discussions as faculty of Aerospace Engineering over the past three years, in the light of our own perspectives as well as the national dialog on engineering education, we all agreed that curricular restructuring should involve not simply "tinkering" with what we had, but real, systemic change. In our discussions, we have attempted to be responsive to three factors in redesigning the curriculum:

- The changed student culture, as discussed above.

- The requirements of ABET 2000 to broaden how we think about engineering, and to be more responsive to the needs of industry and society.

- A stated internal, institutional goal of reducing the number of hours for graduation in largehour programs like engineering.

Considering those three factors, we developed a set of guidelines for change. The following four principles, that we came to over time, channeled the evolution of the new curriculum:

1. A student should be able to graduate in four years without taking enormous overloads or attending summer sessions; we want the quarterly load to be light enough to allow students time to think and learn, not just stagger from midterm to midterm.

2. There should be more flexibility built into the program, allowing electives from a wide variety of areas, from aerodynamics to cockpit design. 
3. The 'engineering science core' must be completed by the end of the third year.

4. Learning should be integrated across the curriculum, utilizing design- or research-related activities to focus students on problem-solving. Comprehensive design activities mimicking real-world scenarios should be woven through the curriculum.

Criterion 4 is responsive to the problems with integration of material across course boundaries, and item 3 really allows the flexibility that makes item 2 possible.

With these four principles in place and agreed to, we found several direct corollaries.

- The total number of quarter hours for graduation should be about 180 , in agreement with what the Provost of the university has suggested. Such a number is also consistent with the results of a survey of the numbers of hours to graduation for aerospace programs at peer universities.

- No student should be enrolled in more than 4 courses total in any quarter.

- The senior year should allow students to explore their own interests through a set of electives.

\section{The Structure of the New Curriculum}

Ultimately, we came to the structure shown graphically in Figure 1, with a tabular listing of courses in Table 1. Much of the first year and part of the second are taken up with science and mathematics, with some specific engineering core material on statics, dynamics, materials and strength of materials in the second year. In addition, thermodynamics as a fundamental discipline underlying much of what we do is taught in the second year. The teaching of differential equations is shared by mathematics and engineering faculty, to help emphasize the applications of those methods to modeling and solving real engineering problems.

The third year, containing the Engineering Science Core, is the heart of the curriculum, and as such involves courses in four disciplines: Aerodynamics/fluid mechanics, Dynamics and systems engineering, Structures and Propulsion/power. Introduction to control is implicit in the systems engineering material, with dedicated control courses left for technical electives in the final year.

Determination to package the material differently has been a constant throughout the entire process of construction of the sophomore and particularly the junior-level courses, in the sense of integrating learning vertically across the curriculum, thereby avoiding the 'modularization' of the material from perspective of the student. To that end, we decided on the following strategy:

- Add a one-hour course to each quarter of the third year, to be called "Systems Integration". This course meets once a week for two hours (as a 'lab'), with all two or three professors, from all of the junior-level core courses for that quarter. One of those two or three professors is to be the Team Leader in a comprehensive design exercise that is expected to span the 
entire third year. We are arranging for aerospace engineers from industry to participate in the teaching of this course sequence, in collaboration with the department's faculty. The students are to be divided into teams, in order to develop students' skills in group interactions. The primary point in the Systems Integration course sequence is not to complete a design per se, which comes in a 3-course sequence in the fourth year, but instead to provide a unifying theme onto which specific uses of the core material may be attached. A total systems perspective is to be instilled in the students as the interaction of disciplines emerges in the process. Oral and written communications skills will be emphasized, as the groups present their ideas to the entire class, and as they submit certain written assignments, like progress reports, throughout the year.

- Generate a comprehensive design problem and tasks, to be used in the Systems Integration sequence in a given year, during meetings in the preceding spring quarter. All department faculty and Design Associates from industry will participate. The exercise is to involve aspects of a complete aerospace system, and to be comprehensive enough to allow relevant input to the design process from elements of all courses in the third-year core.

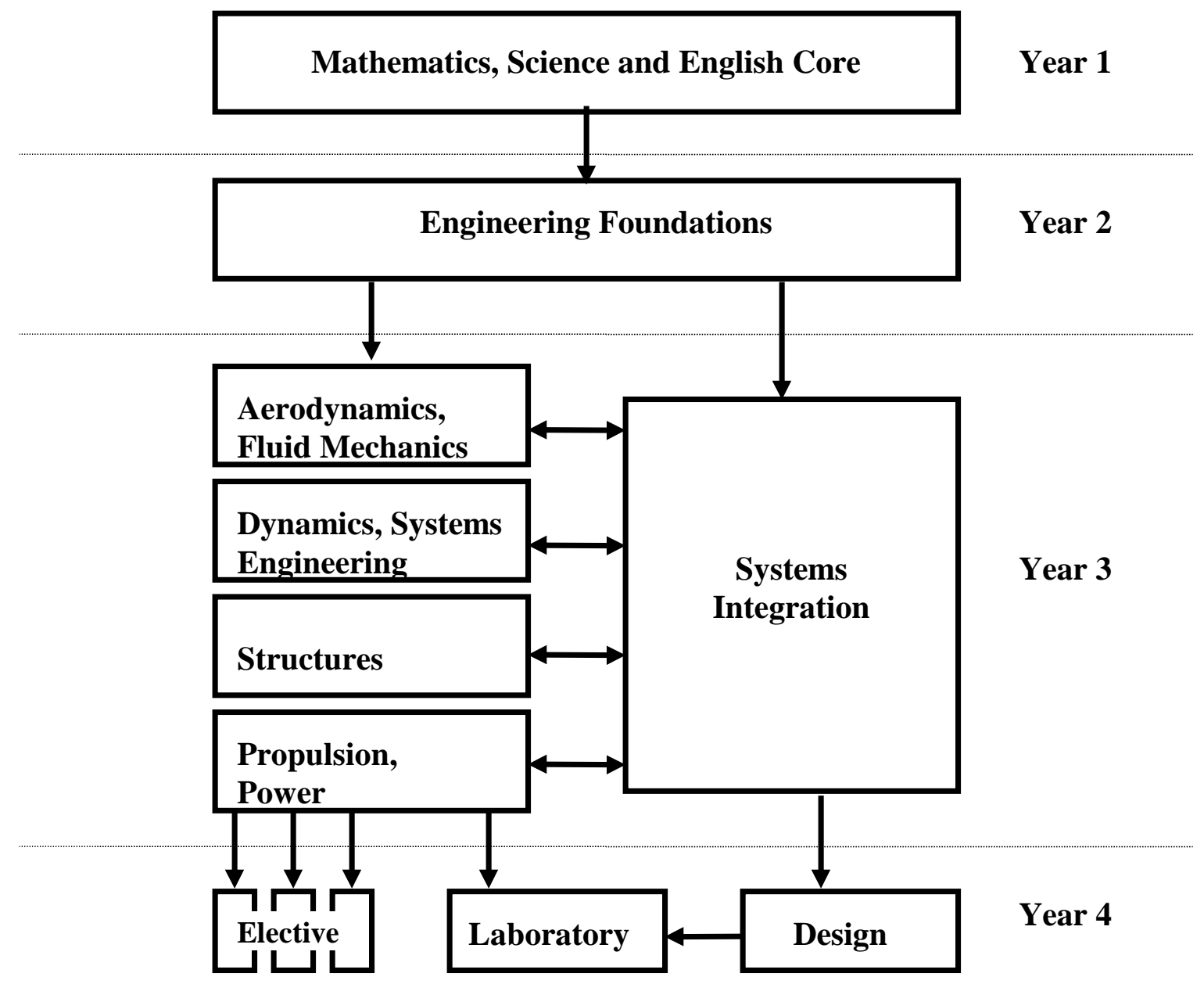

Figure 1. Schematic of the New Curriculum 
One of the goals of the Systems Integration course sequence is motivational, to open the eyes of the students to the character of the design process. We want it to be fun! To engage the students in the process, we have decided to minimize grade anxiety by assigning a "progress" grade only for the first two quarters, with a letter grade for all three hours to be given at the end of the spring quarter course.

Design, laboratories and specialization are the themes of the fourth and final year. A comprehensive capstone design sequence runs the entire final year. That is possible for two reasons: (1) All of the core material is completed in the prior year, and, (2), the students will have experienced systems methodologies in that third year. Included in the capstone design will be mission analysis, preliminary design, and some detail design, using computational and graphical software, to allow the students to acquire skills that they will utilize in their careers. Student laboratories are included throughout the entire senior year as well, with focus on fluids, structures, controls and propulsion during differing portions of the year. Because of the yearlong design sequence, there is potential for connecting some elements of a vehicle configuration to an experimental design and implementation. Indeed, our long-term goal is to transition fully to a project-based laboratory experience, once the necessary resources are secured.

Allowing students to choose three 3-hour technical electives in the senior year creates an opportunity for specialization that was not available in our previous curriculum, which permitted only one technical elective. The three electives may be chosen from a number taught in the department, in advanced aerodynamics, rocket propulsion, control theory, orbital mechanics, structural dynamics, or from suitable courses in other departments. This approach enables students to broaden their education by selecting some courses that are not "technical" in the traditional sense, but are still relevant for engineering. Such courses could be in areas like human factors or cognitive science. This feature of the new curricular structure allows us the flexibility necessary to evolve the program in new directions as the field changes in the future.

\section{Conclusions}

The evolution of our new curriculum for aeronautical and astronautical engineering has been sometimes exciting and sometimes agonizing. In reducing the total number of quarter hours for graduation from 201 to 186, and including more systems methodology and design, we have had to eliminate traditional topics from every discipline. We have attempted to ask the hard questions, to determine, (a), what are the essential concepts that all undergraduates should learn, (b), what may be relegated to undergraduate electives or to graduate courses, and, (c), what is dated or arcane and needs to be discarded. Simultaneous with those three considerations has been the crucial need to incorporate new emphases on systems and design. We believe we have struck a reasonable balance, so that, on the one hand, students who intend to pursue graduate degrees are not without the rigorous background they need; and, on the other hand, students who move immediately into the world of work will have more of those skills required in that sector. 
The move toward department curricular change in Aeronautical and Astronautical Engineering here at The Ohio State University came at a time of national ferment on engineering education, and as ABET 2000 emerged. In the detailed course objectives and syllabi, we are taking actions to assure that we conduct the courses with relevant ABET 2000 criteria integrated into their structure. Such criteria are especially evident in the new courses in systems integration and design.

The changes discussed herein provide us, we believe, with a structure that will better equip our students with the basic engineering fundamentals that will serve them not only in the first five years out of school, but in the last ten years of their careers as well; but will at the same time make better connections of that material for design of aerospace vehicles and for service to society.

\section{References}

1 Postman, N. Amusing Ourselves to Death, Penguin Press, New York, 1985.

2 McMasters, J. H. \& Ford, S. D. “An Industry View of Enhancing Design Education”, AIAA 90-3259. AIAA,AHS/ASEE Aircraft Design and Operation Meeting, Dayton, OH, September, 1990.

3 Dym, C. L. Engineering Design: A Synthesis of Views, Cambridge University Press, New York, 1994.

4 McMasters, J. H. \& Match, L. A. "Desired Attitudes of an Engineering Graduate - An Industry Perspective", AIAA Paper 96-2241, AIAA Advanced Measurement and Ground Testing Technology Conference, New Orleans, June, 1996.

5 "Michigan Curriculum 200: Proposed Changes to the Undergraduate Curriculum", Report to the Undergraduate Curriculum Task Force, College of Engineering University of Michigan,1996.

6 Engineering Criteria 2000, Engineering Accreditation Commission of The Accreditation Board for Engineering and Technology, 1996.

7 Crawley, E. F., Greitzer, E. M., Widnall, S. E., Hall, S. R., McManus, H. L., Hansman, J. R., Shea, J. F. \& Landahl, M. "Reform of the Aeronautics and Astronautics Curriculum at MIT", Journal of Engineering Education 83, 74, 1994.

8 Akin, D. L, Barlow, J. B. \& Schmidt, D. K. "Designing an Aerospace Engineering Curriculum for the Next Century: Experiences at the University of Maryland", presented at the ASEE Annual Conference, Session 0402, 1994.

HAYRANI A. ÖZ

Prof. Öz holds a B.S. degree from Bogazici University, Türkiye, an M.S. degree from The University of Minnesota, and a Ph.D from Virginia Polytechnic Institute and State University. He is a Professor, and Aerospace Section Head, in Aeronautical and Astronautical Engineering at The Ohio State University. His expertise is in structural dynamics, dynamics and control theory, aeroelasticity, astronautics, and design .

MICHAEL R. FOSTER

Prof. Foster holds an S.B. and an S.M. degree from Massachusetts Institute of Technology, and a Ph.D. from California Institute of Technology. He is a Professor of Aeronautical and Astronautical Engineering at The Ohio State University. He teaches courses in fluid mechanics and applied mathematics. His research is in theoretical approaches to geophysical fluid dynamics, hydrodynamic stability, boundary layers and directional solidification. 


\begin{tabular}{|l|l|}
\hline & \multicolumn{1}{|c|}{ Course Title } \\
\hline \hline First Year 48 Hours & Mathematics, Science and English \\
\hline Math 151-153 & Calculus and Analytic Geometry \\
Physics 131-133 & Mechanics, Electromagnetism, Modern Physics \\
Chemistry 121 & General Chemistry \\
Eng Graphics 166, 167 & $\begin{array}{l}\text { Engineering Graphics and Programming } \\
\text { First Writing Course }\end{array}$ \\
\hline \hline Second I Year 47 Hours & Engineering Foundations \\
\hline Math 254 & Multivariable Calculus \\
Math 571 & Linear Algebra for Applications I \\
Chemistry 125 & Chemistry for Engineers \\
Eng Mech 220 & Statics and Strength of Materials \\
Eng Mech 430 & Dynamics \\
MatSci 405 & Introduction to Materials Science and Engineering \\
Aero Eng 200 & Introduction to Aerospace Engineering I \\
Aero Eng 201 & Introduction to Aerospace Engineering II \\
Aero Eng 405 & Thermodynamics \\
Aero Eng 480 & Differential Equations and Engineering Applications \\
English II & Second Writing Course \\
\hline \hline Third Year 45 Hours & Engineering Science Core; Systems \\
\hline ISE 504 & Engineering Economic Analysis \\
Aero Eng 542 & Flight Vehicle Structures I \\
Aero Eng 530 & One-dimensional Gasdynamics \\
Aero Eng 520 & Flight Vehicle Dynamics \\
Aero Eng 512 & Systems Integration I \\
\hline Math 572 & Linear Algebra for Applications II \\
Aero Eng 543 & Flight Vehicle Structures II \\
Aero Eng 560 & Fundamentals of Aerodynamics \\
Aero Eng 521 & Linear Systems Engineering \\
Aero Eng 513 & Systems Integration II \\
\hline Elec Eng 300/309 & Electrical Circuits with Laboratory \\
Aero Eng 570 & Viscous Flow \& Heat Transfer \\
Aero Eng 550 & Propulsion \\
Aero Eng 514 & Systems Integration III \\
\hline \hline Fourth Year 23 Hours & Capstone Design, Laboratory and Specialization \\
\hline Aero 510.01-.03 & Laboratory \\
Design I, II, III & Integrated Design \\
Electives I, II, III & Taken in various specialty areas \\
\hline \hline Other courses 23 Hours & 5 courses in humanities and social science \\
\hline Total Hours & $\mathbf{1 8 6}$ \\
\hline
\end{tabular}

Table 1. Courses for the New Curriculum 